\title{
Ponto de Murcha Permanente de Soja, Feijão e Plantas DANINHAS $^{1}$
}

\author{
Permanent Wilting Point of Soybean, Bean and Weeds \\ PROCÓPIO, S.O. ${ }^{2}$, SANTOS, J.B. ${ }^{3}$, SILVA, A.A. ${ }^{4}$, DONAGEMMA, G.K. ${ }^{5}$ e MENDONÇA, E.S. ${ }^{6}$
}

RESUMO - Objetivou-se neste trabalho determinar o ponto de murcha permanente das culturas da soja e do feijão e de plantas daninhas de grande ocorrência nas áreas agrícolas do Brasil. Os tratamentos foram constituídos por seis espécies, sendo duas cultivadas, Glycine max e Phaseolus vulgaris, e quatro de plantas daninhas - Euphorbia heterophylla (plantas suscetiveis e resistentes a herbicidas inibidores de ALS), Bidens pilosa e Desmodium tortuosum-, com duas épocas de indução de estresse hídrico (pré-florescimento e início do enchimento de grãos). Os solos contidos nos vasos foram mantidos próximos a $80 \%$ da capacidade de campo até as épocas predeterminadas para o início do estresse hídrico. A partir desta etapa não foram mais molhadas e, ao primeiro sinal de murcha das espécies, no final do dia, os respectivos vasos foram transferidos para câmara escura, com umidade relativa do ar próxima a $100 \%$, para constatação do não-retorno definitivo da turgidez [ponto de murcha permanente (PMP)]. Nesse ponto, foi coletada uma amostra (sem raizes) do solo para a determinação do teor de água pelo método gravimétrico. A partir dos valores de umidade determinados nas amostras de solo, para cada tratamento avaliado, caracterizou-se, por meio da curva de retenção de umidade do solo, o potencial da água no PMP. Na fase de enchimento de grãos, o PMP das plantas de B. pilosa ocorreu quando o potencial de retenção de água do solo era mais negativo em relação às outras espécies avaliadas, demonstrando que, neste estádio de desenvolvimento, esta espécie apresenta maior eficiência na extração de água do solo e, conseqüentemente, maior potencial competitivo do que as outras avaliadas.

Palavras-chave: Glycine max, Phaseolus vulgaris, competição, água, déficit hídrico.

\begin{abstract}
This work aimed to determine the permanent wilting point of soybean, and bean crops and weeds of high occurrence in Brazilian agricultural areas. The treatments consisted of two crops, Glycine max and Phaseolus vulgaris, and four weed species: Euphorbia heterophylla (susceptible to ALS - inhibiting herbicides), Euphorbia heterophylla (resistant to ALS - inhibiting herbicides), Bidens pilosa and Desmodium tortuosum, with two water stress induction times (pre-flowering and grain filling onset). The soils in the pots were kept close to $80 \%$ of field capacity until the pre-determined water stress times were achieved. From this stage on the end of the day, the pots were transferred into a dark chamber with relative air humidity close to $100 \%$, to confirm turgidity definitive no- return permanent wilting point (PWP)]. A sample of the soil was collected (without roots) to determine water moisture content using the gravimetric method. Based on the soil moisture values determined for each appraised treatment, soil water potential in PWP was determined by soil moisture retention curve. At the grain filling stage, PWP for B. pilosa occurred when soil water potential was more negative than in the other appraised species, showing that at this developmental stage, B. pilosa presents a greater efficiency in soil water extraction, and, consequently, greater competitive potential than the other appraised species.
\end{abstract}

Key words: Glycine max, Phaseolus vulgaris, competition, water, and water deficit.

Recebido para publicação em 12.8.2003 e na forma revisada em 5.3.2004.

2 Prof. do Dep. de Agronomia, Universidade Federal de Pelotas - UFPEL, Pelotas-RS. ${ }^{3}$ Doutorando, Dep. de Fitotecnia da Universidade Federal de Viçosa - UFV, 36570-000 Viçosa-MG; ${ }^{4}$ Prof. do Dep. de Fitotecnia da UFV; ${ }^{5}$ Doutorando, Dep. de Solos da UFV; ${ }^{6}$ Prof. do Dep. de Solos da UFV. 


\section{INTRODUÇÃO}

Em várias situações de cultivo, a água tem-se mostrado o recurso mais limitante ao crescimento e à produtividade das culturas (Begg \& Turner, 1976). O volume explorado pelo sistema de raízes, a eficiência no uso da água e a capacidade de extração da água do solo determinam a capacidade competitiva de uma planta por esse recurso. Características morfológicas e fisiológicas das plantas determinam suas habilidades competitivas pela água do solo (Griffin et al., 1989). Algumas características, como a maior extensão do sistema radicular, a menor suscetibilidade às intempéries climáticas, como veranico e geadas, e a maior capacidade de produção e liberação de substâncias químicas com propriedades alelopáticas têm sido consideradas como ferramentas destas plantas na competição com as espécies cultivadas (Silva et al., 2000).

A partir do trabalho de Briggs \& Shantz (1912), foi estabelecido que o ponto de murcha permanente ocorre quando a tensão de água no solo é de, aproximadamente, $1.500 \mathrm{kPa}$, o que foi considerado como "conceito das 15 atmosferas", cuja determinação é feita utilizando-se como planta indicadora o girassol. Todavia, vários pesquisadores têm observado que a umidade no PMP pode variar para diferentes espécies de plantas, estádio de desenvolvimento, condições do ambiente e tipo de solo (Slatyer, 1957; Loomis et al., 1960; Sykes, 1969). Este último autor, determinando o potencial de retenção da água no solo no PMP, em dois diferentes solos, encontrou valores variando de 700 a $1.100 \mathrm{kPa}$ para girassol, 1.050 a $1.610 \mathrm{kPa}$ para Nicotiana attenuata, 1.450 a $2.140 \mathrm{kPa}$ para milho, $3.470 \mathrm{kPa}$ para Cassia fasciculata e 2.050 a $3.860 \mathrm{kPa}$ para Agropyron intermedium. Esses resultados contrariam o conceito de que a murcha permanente é independente da espécie e não varia com as características do solo.

Algumas espécies de plantas daninhas são mais competitivas quando o nível de água do solo é ótimo (Hibiscus trionum e Ambrosia artemisifolia), e outras, quando ocorre déficit hídrico (Sorghum halepense, Amaranthus retroflexus e Senna obtusifolia) (Griffin et al., 1989). Há variações na capacidade competitiva entre plantas daninhas e milho e sorgo, quando cultivadas em solo com diferentes niveis de água (Wise \& Van Diver, 1970). Staniforth (1958) constatou reduções na produtividade da soja em áreas infestadas com Setaria glauca quando a umidade do solo foi adequada; no entanto, a competição com esta espécie promoveu déficit hídrico em quase toda a fase reprodutiva da cultura.

Biótipos de Euphorbia heterophylla resistentes aos herbicidas inibidores da ALS foram identificados em lavouras de soja nos Estados do Rio Grande do Sul, Paraná e Mato Grosso do Sul, onde esses produtos são empregados há vários anos (Vargas et al., 1999). Alguns trabalhos mostraram não haver diferença competitiva entre os biótipos sensiveis e resistentes de leiteiro quanto a morfologia, duração do ciclo e potencial de competição com a cultura da soja (Vargas et al., 1999; Vidal \& Trezzi, 2000). Procópio et al. (2002) verificaram que biótipos de $E$. heterophylla, sensiveis e resistentes a herbicidas inibidores de ALS, não diferiram na capacidade competitiva para velocidade de emergência, duração do ciclo e eficiência na utilização da água durante o ciclo. Contudo, ainda há grande carência de informações sobre a ecofisiologia de biótipos de plantas daninhas resistentes a determinados herbicidas, pois a presença delas nas lavouras do País é cada vez maior.

O objetivo deste trabalho foi determinar o ponto de murcha permanente das plantas da soja e do feijão e de plantas daninhas de grande ocorrência nas áreas agrícolas do Brasil, visando conhecer a capacidade de extração de água dessas espécies em dois períodos fenológicos e sob estresse hídrico.

\section{MATERIAL E MÉTODOS}

O experimento foi conduzido em casa de vegetação, no período de agosto a novembro de 2002.

O delineamento experimental foi o de blocos ao acaso, em esquema fatorial $6 \times 2$, constituído por seis espécies - sendo duas cultivadas: soja (cv. Capinópolis) e feijão (cultivar Pérola), e as espécies de plantas daninhas Euphorbia heterophylla (leiteiro - suscetivel a herbicidas inibidores de ALS), Euphorbia heterophylla (leiteiro - resistente a herbicidas inibidores de ALS), Bidens pilosa (picão-preto) 
e Desmodium tortuosum (carrapicho-beiço-deboi) -, com duas épocas de indução de estresse hídrico (pré-florescimento e início do enchimento de grãos), totalizando 12 tratamentos, com três repetições.

As plantas foram cultivadas em vasos plásticos, contendo $4 \mathrm{~kg}$ de solo (Argissolo Vermelho-Amarelo) cada. A amostra de solo foi destorroada, homogeneizada, secada ao ar e passada em peneira de malha de $2 \mathrm{~mm}$. Foi realizada a caracterização física e química do substrato utilizado (Tabela 1), com base na metodologia descrita pela Embrapa (1997). Foi estabelecida a curva de retenção de água do solo nas duas épocas de indução do estresse hídrico.

Antes do preenchimento dos vasos, procedeu-se à adubação do substrato, sendo adicionados $500 \mathrm{~kg} \mathrm{ha}^{-1}$ da fórmula 04-14-08, e correção da acidez com $4 \mathrm{t} \mathrm{ha}^{-1}$ de calcário dolomítico. Em cada vaso foram colocadas cinco sementes, para as culturas, ou 20 sementes, para as espécies de plantas daninhas. Quando as plantas estavam com dois pares de folhas completas, foi realizado desbaste, deixando-se apenas uma planta por vaso.

Os substratos contidos nos vasos foram mantidos próximos a $80 \%$ da capacidade de campo, por meio de três irrigações diárias, até as épocas predeterminadas para o início do estresse hídrico. A partir desta etapa, ao primeiro sinal de murcha das plantas, no final do dia, os respectivos vasos eram transferidos para câmara escura com umidade relativa do ar próxima a $100 \%$. Ao amanhecer era observado o retorno ou não da turgidez de cada planta, adotando-se o critério de que, se pelo menos uma folha se recuperasse, o vaso retornava à casa de vegetação e continuava sendo mantido sem irrigação, e assim sucessivamente, até o não-retorno definitivo da turgidez [ponto de murcha permanente (PMP)]. Nesse ponto, foi coletada uma amostra do solo (sem raízes), pesada em balança de precisão (Cassel $\&$ Nielsen, 1986) e imediatamente levada para estufa, a $105{ }^{\circ} \mathrm{C}$, por 24 horas. A seguir, era novamente pesada, para determinação da umidade da amostra em base gravimétrica. A partir dos teores de água nas amostras do substrato para cada tratamento avaliado, determinou-se, por meio da curva de retenção de água, o potencial de retenção desta no PMP. Também, quando da ocorrência do PMP, as plantas eram coletadas, secadas em estufa a $75^{\circ} \mathrm{C}$ por 72 horas, ocasião em que se determinou e a biomassa seca.

Terminada a coleta e tabulação dos dados, estes foram submetidos à análise de variância e, quando pertinentes, as médias foram comparadas pelo teste de Tukey a $5 \%$ de probabilidade.

\section{RESULTADOS E DISCUSSÃO}

Todas as espécies avaliadas apresentaram, em média, três dias entre a indução do estresse e o ponto de murcha permanente. Observouse que, quando o estresse hídrico foi induzido no estádio de pré-florescimento, as plantas de $B$. pilosa tiveram sua murcha permanente em solo mais úmido que as demais espécies, apenas não diferindo das plantas de soja (Tabela 2 e Figura 1), demonstrando a menor

Tabela 1 - Composição físico-química da camada arável $(0-20 \mathrm{~cm})$ do solo Argissolo Vermelho-Amarelo utilizado no experimento

\begin{tabular}{|c|c|c|c|c|c|c|c|c|c|c|}
\hline \multicolumn{11}{|c|}{ Análise granulométrica $\left(\mathrm{dag} \mathrm{kg}^{-1}\right)$} \\
\hline \multicolumn{2}{|c|}{ Argila } & \multicolumn{2}{|r|}{ Silte } & \multicolumn{2}{|c|}{ Areia fina } & \multicolumn{2}{|c|}{ Areia grossa } & \multicolumn{3}{|c|}{ Classificação textural } \\
\hline \multicolumn{2}{|c|}{60} & \multicolumn{2}{|r|}{07} & \multicolumn{2}{|r|}{11} & \multicolumn{2}{|c|}{22} & \multicolumn{3}{|c|}{ Argiloso } \\
\hline \multicolumn{11}{|c|}{ Análise Química } \\
\hline \multirow{2}{*}{$\begin{array}{c}\mathrm{pH} \\
\mathrm{H}_{2} \mathrm{O}\end{array}$} & $\mathrm{P}$ & $\mathrm{K}^{+}$ & $\mathrm{H}+\mathrm{Al}$ & $\mathrm{Al}^{3+}$ & $\mathrm{Ca}^{2+}$ & $\mathrm{Mg}^{2+}$ & $\mathrm{CTC}_{\text {total }}$ & $\mathrm{V}$ & $\mathrm{M}$ & $\mathrm{MO}$ \\
\hline & \multicolumn{2}{|c|}{$\left(\mathrm{mg} \mathrm{dm}^{-3}\right)$} & \multicolumn{5}{|c|}{$\left(\mathrm{cmol}_{\mathrm{c}} \mathrm{dm}^{-3}\right)$} & \multicolumn{2}{|c|}{$(\%)$} & $\left(\right.$ dag kg $\left.^{-1}\right)$ \\
\hline 4,2 & 1,6 & 34 & 8,32 & 1,56 & 0,28 & 0,15 & 8,84 & 5,9 & 75 & 3,81 \\
\hline
\end{tabular}

* Análises realizadas nos Laboratórios de Análises Físicas e Químicas de Solo do Departamento de Solos da UFV, segundo a metodologia descrita pela Empresa Brasileira de Pesquisa Agropecuária-EMBRAPA (1997). 
capacidade de extração de água do solo das plantas de picão-preto nesse estádio fenológico. $E$. heterophylla (suscetivel e resistente), D. tortuosum e feijão foram as espécies em que o momento de murcha permanente ocorreu quando o solo tinha o menor teor de água, diferindo do ocorrido com $B$. Pilosa. No entanto, apenas os resultados das plantas de $E$. heterophylla suscetiveis aos herbicidas inibidores da ALS diferiram daqueles verificados para as plantas de soja (Tabela 2).
O teor de água no solo no PMP das espécies avaliadas, induzido após o início do enchimento de grãos, foi significativamente menor para as plantas de $B$. pilosa em relação ao das demais espécies (Tabela 2), indicando que, nesse estádio, plantas de $B$. pilosa conseguem absorver água mais fortemente retida no solo, o que pode significar maior tolerância à seca, em relação às outras espécies avaliadas. A competição entre Sorghum halepense (McWhorter \& Hartwig, 1972) ou Amaranthus retroflexus

Tabela 2 - Umidade do solo e biomassa seca de algumas espécies vegetais no ponto de murcha permanente, em dois estádios fenológicos. Viçosa-MG, 2002

\begin{tabular}{|l|c|c|c|c|}
\hline \multirow{2}{*}{ Espécie vegetal } & \multicolumn{2}{|c|}{ Umidade do solo (\%) } & \multicolumn{2}{c|}{ Biomassa seca total $\left(W_{t}\right)\left(\mathrm{g}\right.$ planta $\left.{ }^{-1}\right)$} \\
\cline { 2 - 5 } & Pré-florescimento & $\begin{array}{c}\text { Início do enchimento } \\
\text { de grãos }\end{array}$ & Pré-florescimento & $\begin{array}{c}\text { Início do enchimento } \\
\text { de grãos }\end{array}$ \\
\hline Phaseolus vulgaris & $15,66 \mathrm{ab} \mathrm{A}$ & $16,65 \mathrm{~b} \mathrm{~B}$ & $4,08 \mathrm{c} \mathrm{B}$ & $30,29 \mathrm{c} \mathrm{A}$ \\
\hline Glycine max & $16,18 \mathrm{bc} \mathrm{A}$ & $16,27 \mathrm{~b} \mathrm{~A}$ & $20,45 \mathrm{a} \mathrm{B}$ & $110,86 \mathrm{a} \mathrm{A}$ \\
\hline Euphorbia heterophylla (resistente*) & $15,40 \mathrm{ab} \mathrm{A}$ & $16,53 \mathrm{~b} \mathrm{~B}$ & $0,91 \mathrm{~d} \mathrm{~B}$ & $26,83 \mathrm{~d} \mathrm{~A}$ \\
\hline Euphorbia heterophylla (suscetível*) & $14,78 \mathrm{a} \mathrm{A}$ & $16,67 \mathrm{~b} \mathrm{~B}$ & $0,83 \mathrm{~d} \mathrm{~B}$ & $26,57 \mathrm{~d} \mathrm{~A}$ \\
\hline Bidens pilosa & $17,47 \mathrm{c} \mathrm{B}$ & $12,17 \mathrm{a} \mathrm{A}$ & $1,08 \mathrm{~d} \mathrm{~B}$ & $44,12 \mathrm{~b} \mathrm{~A}$ \\
\hline Desmodium tortuosum & $15,60 \mathrm{ab} \mathrm{A}$ & $16,86 \mathrm{~b} \mathrm{~B}$ & $12,54 \mathrm{~b} \mathrm{~B}$ & $31,45 \mathrm{c} \mathrm{A}$ \\
\hline
\end{tabular}

* Biótipo resistente e suscetível aos herbicidas inibidores da enzima ALS.

Médias seguidas de letras iguais, minúsculas na coluna e maiúsculas na linha, não diferem pelo teste de Tukey a 5\% de probabilidade.

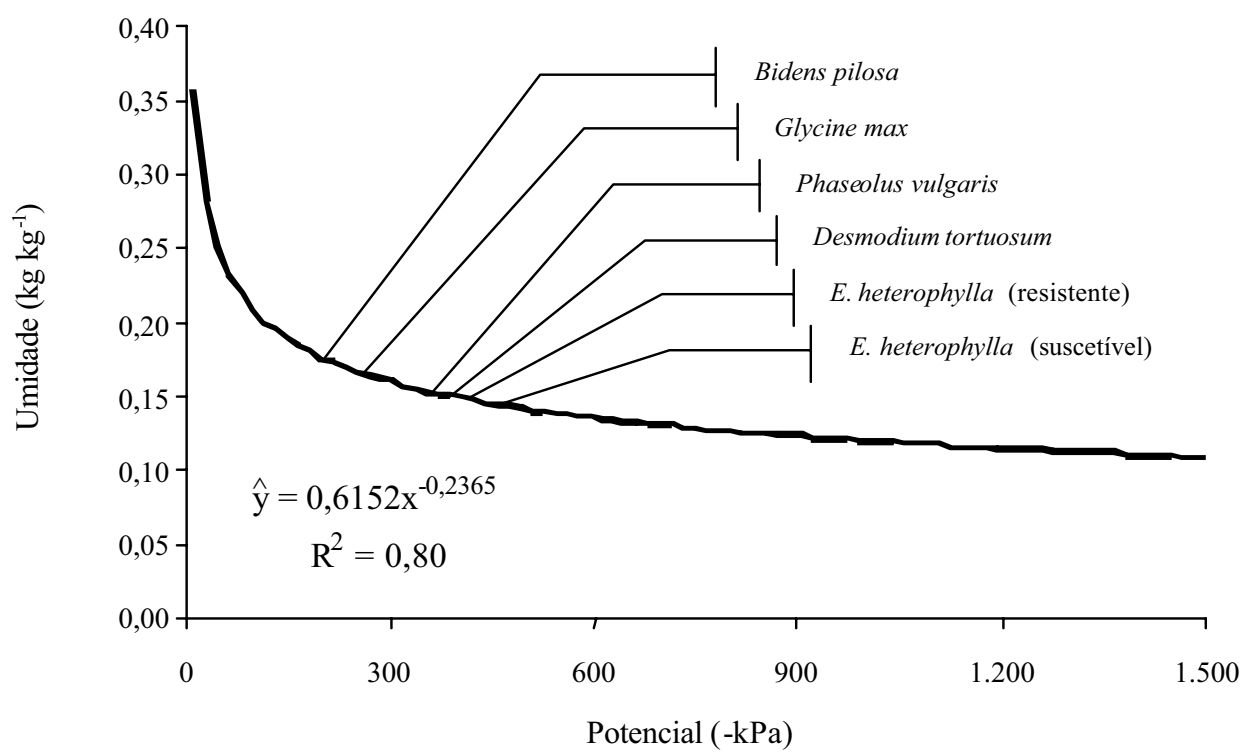

Figura 1 - Potencial hídrico do solo no ponto de murcha permanente para as espécies: soja, feijão, E. heterophylla (suscetível e resistente aos herbicidas inibidores da enzima ALS), Bidens pilosa e Desmodium tortuosum, com indução do estresse hídrico no início do florescimento. 
(Orwick \& Schreiber, 1979) e a soja é intensificada com o déficit hídrico.

A umidade do solo no PMP induzido após o período de início do enchimento de grãos foi maior do que a observada na fase de pré-florescimento para as plantas de feijão, $E$. heterophylla (suscetivel ou resistente) e D. tortuosum (Tabela 2), demonstrando menor tolerância à seca no enchimento de grãos. Não foi observada mudança no comportamento da soja no que se refere à extração de água do solo. B. pilosa foi a única espécie que demonstrou maior eficiência na retirada de água do solo quando se encontrava no período de enchimento de grãos (Tabela 2) e, nesta fase, foi a planta que apresentou a segunda maior biomassa. Todavia, não houve relação entre a capacidade de absorção de água do solo e a biomassa formada pelas espécies. Plantas jovens de Anthemis cotula não apresentaram redução da biomassa seca quando o potencial de retenção de água no solo foi mantido em $-250 \mathrm{kPa}$ (Gealy et al., 1994).

Tabela 3 - Potencial hídrico do solo cultivado com diferentes espécies, no ponto de murcha permanente, em dois estádios fenológicos. Viçosa-MG, 2002

\begin{tabular}{|c|c|c|}
\hline \multirow{2}{*}{ Espécie vegetal } & Pré-florescimento & Início do enchimento de grãos \\
\hline & \multicolumn{2}{|c|}{ Potencial $(\mathrm{kPa})$} \\
\hline Phaseolus vulgaris & 325,33 a A & $251,57 \mathrm{~b} \mathrm{~A}$ \\
\hline Glycine $\max$ & 283,41 a $\mathrm{A}$ & $283,01 \mathrm{~b} \mathrm{~A}$ \\
\hline Euphorbia heterophylla (resistente*) & 351,79 a A & $259,21 \mathrm{~b} \mathrm{~A}$ \\
\hline Euphorbia heterophylla (suscetível*) & 419,11 a A & $250,20 \mathrm{~b} \mathrm{~B}$ \\
\hline Bidens pilosa & 206,27 a B & 982,91 a A \\
\hline Desmodium tortuosum & 335,79 a A & 241,72 b A \\
\hline
\end{tabular}

* Biótipo resistente e suscetível aos herbicidas inibidores da enzima ALS.

Médias seguidas de letras iguais, minúsculas na coluna e maiúsculas na linha, não diferem pelo teste de Tukey a 5\% de probabilidade.

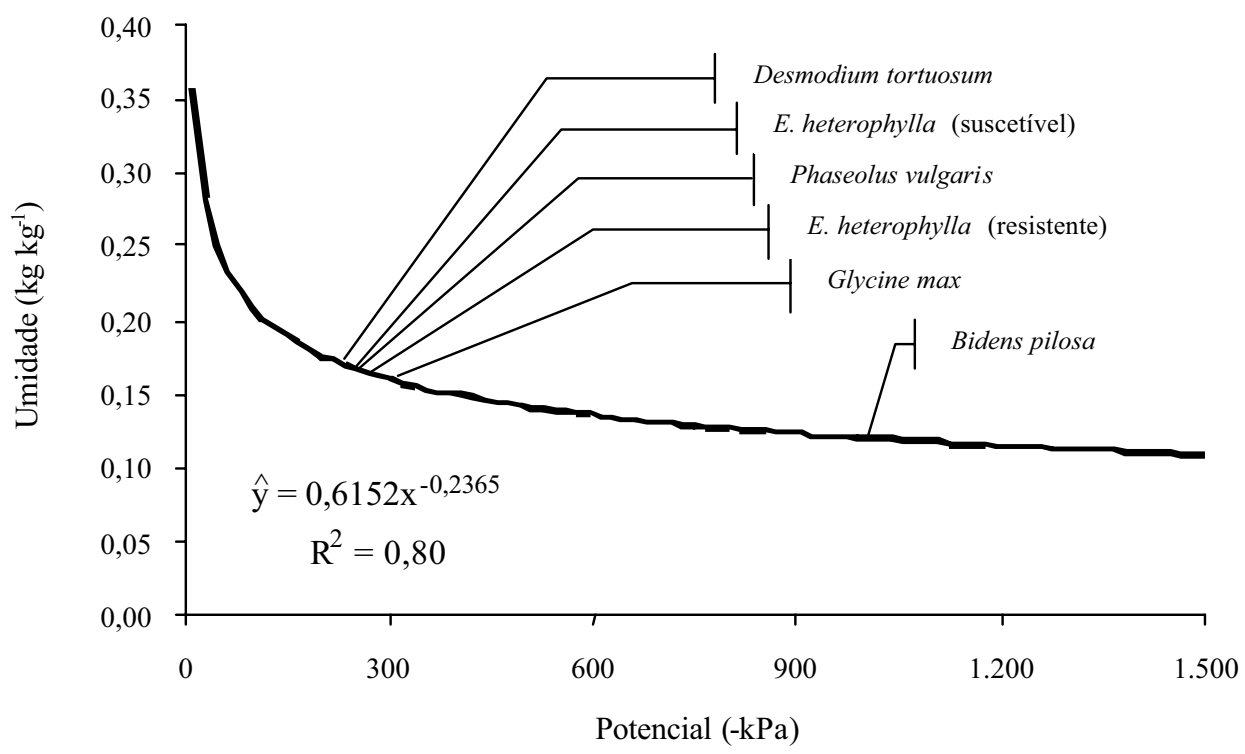

Figura 2 - Potencial hídrico do solo no ponto de murcha permanente para as espécies: soja, feijão, E. heterophylla (suscetível e resistente aos herbicidas inibidores da enzima ALS), Bidens pilosa e Desmodium tortuosum, com indução do estresse hídrico no início do enchimento de grãos. 
$\mathrm{Na}$ fase de pré-florescimento e no PMP de todas as espécies não ocorrreu diferença no potencial hídrico do solo (Tabela 3). Entretanto, o potencial hídrico do solo no PMP induzido após o início do enchimento de grãos foi muito mais negativo no solo onde se cultivou $B$. pilosa (Tabela 3 e Figura 2), reafirmando a maior capacidade desta espécie em absorver a fração da água mais fortemente retida no solo e, conseqüentemente, a sua maior capacidade competitiva, sobretudo em situações de baixa disponibilidade hídrica. Sob condições de estresse hídrico, plantas de Senna obtusifolia reduzem $15 \%$ a mais a matéria seca da soja, em comparação com as crescidas em solo mantido na capacidade de campo (Patterson, 1986), e as de Xanthium pensylvanicum têm vantagens competitivas sobre a soja (Geddes et al., 1979).

O potencial hídrico do solo no PMP onde se cultivou $E$. heterophylla suscetivel aos herbicidas inibidores da ALS foi menor quando o estresse hídrico foi induzido no início do período de enchimento de grãos (Tabela 3), ou seja, a planta murchou nesta fase quando o solo tinha mais umidade que em relação à umidade do solo no PMP observado no préflorescimento. Esse comportamento foi o inverso do observado em $B$. pilosa. O potencial de retenção da água no solo no ponto de murcha permanente verificado para todas as espécies avaliadas foi maior do que o adotado por Briggs \& Shantz (1912), de $1.500 \mathrm{kPa}$, tomando-se por base plantas de girassol.

Pelas informações geradas, pode-se deduzir que em períodos de veranico as plantas de $B$. pilosa, em fase de enchimento de grãos, podem se tornar mais competitivas em lavouras, principalmente de soja ou de feijão, em decorrência da sua maior capacidade na absorção de água do solo. Também, pode-se concluir que $B$. pilosa pode completar seu ciclo mesmo em condições de menor umidade do solo, produzindo propágulos e, conseqüentemente, aumentando o banco de suas sementes na área.

\section{LITERATURA CITADA}

BEGG, S. E.; TURNER, N. C. Crop water deficits. Adv. Agron., v. 28, p. 161-217, 1976.
BRIGGS, L. J.; SHANTZ, H. L. The wilting coefficient for different plants and its indirect determination. Washington: Department of Agriculture, 1912. 83 p. (Bulletin, 230)

CASSEL, D. K.; NIELSEN, D. R. Field capacity and avaliable water capacity. In: KLUTE, A. (Ed.) Methods of soil analysis. Part 1 - Physical and mineralogical methods. 2.ed. Madison: SSSA, 1986. p. 901-926. (Book Series, 5)

EMPRESA BRASILEIRA DE PESQUISA

AGROPECUÁRIA - EMBRAPA. Centro Nacional de Pesquisa de Solos. Manual de métodos de análise de solo. 2.ed. Rio de Janeiro, 1997. 212 p.

GEALY, D. R.; SQUIER, S. A.; OGG Jr., A. G. Soil environment and temperature affect germination and seedling growth of mayweed chamomile (Anthemis cotula). Weed Technol., v. 8, p. 668-672, 1994.

GEDDES, R. D.; SCOTT, H. D.; OLIVER, L. R. Growth and water use by common cocklebur (Xanthium pensylvanicum) and soybeans (Glycine max) under field conditions. Weed Sci., v. 27, p. 206-212, 1979.

GRIFFIN, B. S. et al. The influence of water stress on the physiology and competition of soybean (Glycine max) and florida beggarweed (Desmodium tortuosum). Weed Sci., v. 37, p. $544-551,1989$.

LOOMIS, W. E.; SANTAMARIA, P. R.; GAGE, R. S. Cohesion of water in plants. Plant Physiol., v. 35, p. 300306, 1960

McWHORTER, C. G.; HARTWIG, E. E. Competition of johnsongrass and cocklerbur with six soybean varieties. Weed Sci., v. 20, p. 56-59, 1972.

ORWICK, P. L.; SCHREIBER, M. M. Interference of redroot pigweed and robust foxtail in soybeans. Weed Sci., v. 27 , p. $665-674,1979$.

PATTERSON, D. T. Effects of moisture stress on growth and competitiveness of soybeans and sicklepod. Proc. Weed Sci. Soc., v. 26, p. 59-60, 1986.

PROCÓPIO, S. O. et al. Análise do crescimento e eficiência no uso da água pelas culturas de soja e feijão e por plantas daninhas. Acta Sci., v. 24, p. 1345-1351, 2002.

SILVA, A. A. et al. Controle de plantas daninhas. Brasília: ABEAS, 2000. 260 p.

SLATYER, R. O. The significance of the permanent wilting percentage in studies of plant and soil water relations. Bot. Rev., v. 23, p. 585-636, 1957. 
STANIFORTH, D. W. Soybean-foxtail competition under varying soil moisture conditions. Agron. J., v. 50, p. 13-15, 1958.

SYKES, D. J. Reconsideração do conceito da umidade de murchamento permanente. Turrialba, v. 19, p. 525-530, 1969.

SYKES, D. J.; LOOMIS, W. E. Plant and soil factors in permanent wilting percentages and field capacity storage. Soil Sci., v. 104, p. 162-173, 1969.
VARGAS, L. et al. Resistência de plantas daninhas a herbicidas. Viçosa: Edição dos autores, 1999. 131 p.

VIDAL, R. A.; TREZZI, M. M. Análise de crescimento de biótipos de leiteira (Euphorbia heterophylla) resistentes e susceptível aos herbicidas inibidores da ALS. Planta Daninha, v. 18, p. 427-433, 2000.

WIESE, A. F.; Van DIVER, C. W. Soil moisture effects on competitive ability of weeds. Weed Sci., v. 18, p. 518-519, 1970 